\title{
mSQG equations in distributional spaces and point vortex approximation
}

\author{
Franco Flandoli*and Martin Saal ${ }^{\dagger}$
}

April 17, 2019

\begin{abstract}
Existence of distributional solutions of a modified Surface Quasi-Geostrophic equation (mSQG) is proved for $\mu$-almost every initial condition, where $\mu$ is a suitable Gaussian measure. The result is the by-product of existence of a stationary solution with white noise marginal. This solution is constructed as a limit of random point vortices, uniformly distributed and rescaled according to the Central Limit Theorem 1
\end{abstract}

\section{Introduction}

The Surface Quasi-Geostrophic equation (SQG) in the torus $\mathbb{T}^{2}=\mathbb{R}^{2} / \mathbb{Z}^{2}$ is the equation

$$
\begin{aligned}
& \partial_{t} \theta+u \cdot \nabla \theta=0, \\
& u=\nabla^{\perp} \Lambda^{-1} \theta=\mathcal{R}^{\perp} \theta,
\end{aligned}
$$

where $\Lambda=(-\Delta)^{1 / 2}$ is the square root of the Laplacian, $\nabla^{\perp}=\left(-\partial_{y}, \partial_{x}\right)$ and $\mathcal{R}=\left(\mathcal{R}_{1}, \mathcal{R}_{2}\right)$ is the vector of Riesz-transforms. It has applications in both meteorological and oceanic flows, and it describes the temperature $\theta$ in a rapidly rotating stratified fluid with uniform potential vorticity (see [20], 29], [33] for the geophysical background). In mathematics, it received a lot of attention because of structural similarities to the 3D Euler equations as pointed out in [9] and [10]. The quantity $\nabla^{\perp}$ plays a similar role for SQG as the vorticity does for the 3D Euler equations, and also the level sets of $\theta$ are analogous to vortex lines for 3D Euler equations to mention just some of the similarities of the systems. While for initial data $\theta_{0} \in H^{k}\left(\mathbb{T}^{2}\right)$, the existence of unique local solutions in $C\left([0, T] ; H^{k}\left(\mathbb{T}^{2}\right)\right)$ for $k \geq 3$ is known since those works, the question of global existence of regular solutions

\footnotetext{
* Scuola Normale Superiore di Pisa Classe di Scienze, Pisa, Italy, e-mail: franco.flandoli@sns.it

${ }^{\dagger}$ Department of Mathematics, TU Darmstadt, Schlossgartenstr. 7, 64289 Darmstadt, Germany, e-mail: msaal@mathematik.tu-darmstadt.de

${ }^{1}$ MSC 2010: primary 60H15; secondary 35Q86, 35R60, 76B03.
} 
is still open. The existence of weak solutions $\theta \in L^{\infty}\left((0, \infty) ; L^{2}\left(\mathbb{T}^{2}\right)\right)$ for initial data in $\theta_{0} \in L^{2}\left(\mathbb{T}^{2}\right)$ was first established in [30] and later extended in [25] to the case $\theta_{0} \in L^{p}\left(\mathbb{T}^{2}\right)$ with solutions $\theta \in L^{\infty}\left((0, \infty) ; L^{p}\left(\mathbb{T}^{2}\right)\right)$ when $4 / 3<p<\infty$, but the uniqueness is an open problem. Note that all these results also hold when $\mathbb{T}^{2}$ is replaced by $\mathbb{R}^{2}$. By the technique of convex integration in [3, the non-uniqueness of weak solutions with less regularity than the above ones is established. Due to the properties of the nonlinearity, weak solutions to SQG can be defined even for distributional-valued functions $\theta \in L_{\text {loc }}^{2}\left(\mathbb{R} ; \dot{H}^{-1 / 2}\left(\mathbb{T}^{2}\right)\right)$, and for sufficiently regular $\theta$, the $\dot{H}^{-1 / 2}$-norm is conserved, see [3] and [21] for details. However, in [2] it is shown that uniqueness can be restored by adding random diffusion. A solution theory in Hölder spaces is given in 34, the equation admits a local solution $\theta \in L^{\infty}\left((0, T) ; C^{r}\left(\mathbb{R}^{2}\right) \cap L^{p}\left(\mathbb{R}^{2}\right)\right)$ for initial data $\theta_{0} \in C^{r}\left(\mathbb{R}^{2}\right) \cap L^{p}\left(\mathbb{R}^{2}\right)(r, q>1)$.

While the regularity problem for smooth solutions is unsolved, in the recent work [5] an example for a non-trivial, global smooth solution with finite energy is constructed in the whole space setting. There are few results apart from the whole space $\mathbb{R}^{2}$ or the torus $\mathbb{T}^{2}$. For an open and bounded set $\Omega \subset \mathbb{R}^{d}(d \geq 2)$ with smooth boundary in [11], the global existence of weak solutions $\theta \in L^{\infty}\left((0, \infty) ; L^{2}(\Omega)\right)$ for initial data $\theta_{0} \in L^{2}(\Omega)$ is proved. This case is more difficult because a helpful commutator structure in the nonlinear term is broken by the lack of translation invariance.

We consider here a family of equations that links SQG to the 2D Euler equations, with a smoother velocity field $u$ than in SQG, called modified SQG (mSQG, sometimes it is also referred to as generalized SQG). It reads

$$
\begin{aligned}
& \partial_{t} \theta+u \cdot \nabla \theta=0, \\
& u=\nabla^{\perp} \Lambda^{-1-\epsilon} \theta
\end{aligned}
$$

with $\epsilon \in(0,1)$. For $\epsilon=1$ we obtain the vorticity formulation of the $2 \mathrm{D}$ Euler equations and for $\epsilon=0$ SQG. For the 2D Euler equations the global regularity has been established (see for example [24]), and the above family of equations has been introduced to approach SQG ([7], [13]). As SQG itself this family admits the conservation of the $L^{p}$-norm of solutions for $1 \leq p \leq \infty$. For mSQG we have that similar theorems on the existence of solutions as for SQG hold and some additional results, including the following ones:

1. For initial data in $\theta_{0} \in C^{r}\left(\mathbb{T}^{2}\right)$, there is a local strong solution $\theta \in L^{\infty}\left((0, T) ; C^{r}\left(\mathbb{T}^{2}\right)\right)$ $(r>1)$. The global existence of strong solutions is an open problem for all $\varepsilon \in(0,1]$.

2. For initial data in $\theta_{0} \in L^{p}\left(\mathbb{T}^{2}\right)$, there is a global weak solution $\theta \in L^{\infty}\left((0, \infty) ; L^{p}\left(\mathbb{T}^{2}\right)\right)$ $(p>4 / 3)$.

3. The vortex patch problem, i.e., considering as initial data the characteristic function of a domain with sufficiently smooth boundary (or more generally the sum of characteristic functions) and investigating if the boundary remains smooth, has a unique local solution ([12], [16], [19], 23], [31]). Note that here the existence of a blow-up is known in special cases $([22])$. 
4. A global flow $\psi$ defined on some probability space with values in $C\left([0, T], H^{-2-}\left(\mathbb{T}^{2}\right)\right)$ for mSQG was constructed in [27].

General domains have been studied in [28] and even more singular velocity fields in [6].

From a deterministic perspective, the purpose of this work is to prove existence of solutions of class

$$
\theta \in C\left([0, T] ; H^{-1-}\left(\mathbb{T}^{2}\right)\right)
$$

where $H^{-1-}\left(\mathbb{T}^{2}\right)=\cap_{\epsilon>0} H^{-1-\epsilon}\left(\mathbb{T}^{2}\right)$. Here $H^{s}\left(\mathbb{T}^{2}\right)$ are the classical Sobolev spaces on $\mathbb{T}^{2}$ with integrability order $s$, defined for all real numbers $s$. Opposite to more classical existence results in classes of more regular functions, we may prove solvability in $C\left([0, T] ; H^{-1-}\left(\mathbb{T}^{2}\right)\right)$ only for $\mu$-almost every initial condition $\theta_{0} \in H^{-1-}\left(\mathbb{T}^{2}\right)$, where $\mu$ is a suitable Gaussian measure supported on $H^{-1-}\left(\mathbb{T}^{2}\right)$ but not on $H^{-1}\left(\mathbb{T}^{2}\right)$ and not on the subspace of $H^{-1-}\left(\mathbb{T}^{2}\right)$ of signed measures. Our result on the existence of such a solution is the following.

Theorem 1 For all $\epsilon \in(0,1)$, there exists a probability space $(\Xi, \mathcal{F}, P)$ and a measurable map $\theta .: \Xi \times[0, T] \rightarrow H^{-1-}\left(\mathbb{T}^{2}\right)$ ( $T>0$ arbitrary) with continuous paths such that $\theta$. is a solution to $m S Q G$ in the sense of Definition 9 and $\left(\theta_{t}\right)$ is stationary.

We prove only existence; uniqueness is a major open problem. Our second main result is the approximation of these distributional solutions by the, analogous for mSQG, so called point vortex solutions (we shall use this name also for mSQG below)

$$
\theta(t)=\frac{1}{\sqrt{N}} \sum_{i=1}^{N} \xi_{i} \delta_{x_{t}^{i}}
$$

where the dynamics of the point vortices $x_{t}^{i}$ is given by

$$
\frac{d x_{i}(t)}{d t}=\frac{1}{\sqrt{N}} \sum_{j=1}^{N} \xi_{j} K\left(x_{i}(t)-x_{j}(t)\right), \quad i=1, \ldots, N
$$

with the kernel $K$ and the intensities $\xi_{i}$ specified below. We show that the solution obtained in Theorem 1 can be approximated by the corresponding point vortex system.

Theorem 2 The random point vortex system defined on $(\Xi, \mathcal{F}, P)$ has a subsequence which converges $P$-a.s. to $\theta$ in $C\left([0, T] ; H^{-1-}\left(\mathbb{T}^{2}\right)\right)$.

In some cases it has been proved that point vortices are limits of regular $\theta$-patches (for Euler equations see [15], for a class of mSQG models see [18]), when this occurs it implies that the distributional solutions constructed here are also limits of regular patches.

From a stochastic perspective, we prove existence of a stationary solution of mSQG having marginals with the white noise law, hence supported on $H^{-1-}\left(\mathbb{T}^{2}\right)$. The stationary 
solution is constructed as a weak limit of random point vortices, distributed uniformly in space, with CLT-scaling of intensities.

The results proved here for mSQG model are parallel to those obtained in [15] for 2D Euler equations. Limited to the existence of a stationary solutions with white noise marginal, there is intersection with the results of [27, proved by a different method, along the lines of [1] (based on Galerkin approximation instead of point vortices, which makes less clear the connection with other classes of solutions to the equation). Results on mSQG model and point vortices, in directions different from ours, have been recently proved by [17, [18. For the whole space setting the idea of point vortices for mSQG is sketched in [4].

Last we want to mention that there is a second way to introduce some smoothing into the equation by adding viscosity of the form $\Lambda^{\gamma} \theta$ to the evolution equation for $\theta$ with $\gamma>0$. This dissipative SQG has been investigated by many authors; we refer here to [8] and the references therein for more information.

\section{The nonlinear term in the weak formulation}

\subsection{White noise}

We denote by $\left\{e_{n}\right\}$ the complete orthonormal system in $L^{2}\left(\mathbb{T}^{2}, \mathbb{C}\right)$ given by $e_{n}(x)=e^{2 \pi i n \cdot x}$, $n \in \mathbb{Z}^{2}$. Given a distribution $\omega \in C^{\infty}\left(\mathbb{T}^{2}\right)^{\prime}$ and a test function $\phi \in C^{\infty}\left(\mathbb{T}^{2}\right)$, we denote by $\langle\omega, \phi\rangle$ the duality between $\omega$ and $\phi$ (namely $\omega(\phi)$ ), and we use the same symbol for the inner product of $L^{2}\left(\mathbb{T}^{2}\right)$. We set $\widehat{\omega}(n)=\left\langle\omega, e_{n}\right\rangle, n \in \mathbb{Z}^{2}$, and we define, for each $s \in \mathbb{R}$, the space $H^{s}\left(\mathbb{T}^{2}\right)$ as the space of all distributions $\omega \in C^{\infty}\left(\mathbb{T}^{2}\right)^{\prime}$ such that

$$
\|\omega\|_{H^{s}}^{2}:=\sum_{n \in \mathbb{Z}^{2}}\left(1+|n|^{2}\right)^{s}|\widehat{\omega}(n)|^{2}<\infty .
$$

We use similar definitions and notations for the space $H^{s}\left(\mathbb{T}^{2}, \mathbb{C}\right)$ of complex valued functions. In the space $H^{-1-}\left(\mathbb{T}^{2}\right)=\bigcap_{\epsilon>0} H^{-1-\epsilon}\left(\mathbb{T}^{2}\right)$, we consider the metric

$$
d_{H^{-1-}}\left(\omega, \omega^{\prime}\right)=\sum_{n=1}^{\infty} 2^{-n}\left(\left\|\omega-\omega^{\prime}\right\|_{H^{-1-\frac{1}{n}}} \wedge 1\right) .
$$

Convergence in this metric is equivalent to convergence in $H^{-1-\epsilon}\left(\mathbb{T}^{2}\right)$ for every $\epsilon>0$. The space $H^{-1-}\left(\mathbb{T}^{2}\right)$ with this metric is complete and separable. We denote by $\mathcal{X}:=$ $C\left([0, T] ; H^{-1-}\left(\mathbb{T}^{2}\right)\right)$ the space of continuous functions with values in this metric space; a function is in $\mathcal{X}$ if and only if it is in $C\left([0, T] ; H^{-1-\epsilon}\left(\mathbb{T}^{2}\right)\right)$ for every $\epsilon>0$. The distance in $C\left([0, T] ; H^{-1-}\left(\mathbb{T}^{2}\right)\right)$ is given by $d_{\mathcal{X}}\left(\omega ., \omega^{\prime}\right)=\sup _{t \in[0, T]} d_{H^{-1-}}\left(\omega_{t}, \omega_{t}^{\prime}\right)$, which makes $\mathcal{X}$ a Polish space. 
For $s>0$, the spaces $H^{s}\left(\mathbb{T}^{2}\right)$ and $H^{-s}\left(\mathbb{T}^{2}\right)$ are dual each other. By $H^{s+}\left(\mathbb{T}^{2}\right)$, we shall therefore mean the space $\bigcup_{\epsilon>0} H^{s+\epsilon}\left(\mathbb{T}^{2}\right)$. We will use this notation also in the case of the space $H^{2+}\left(\mathbb{T}^{2} \times \mathbb{T}^{2}\right)$, which is similarly defined.

We start recalling the well known notion of white noise, reviewing some of its main properties used in the sequel.

White noise on $\mathbb{T}^{2}$ is by definition a Gaussian distributional-valued stochastic process $\omega: \Xi \rightarrow C^{\infty}\left(\mathbb{T}^{2}\right)^{\prime}$, defined on some probability space $(\Xi, \mathcal{F}, \mathbb{P})$, such that

$$
\mathbb{E}[\langle\omega, \phi\rangle\langle\omega, \psi\rangle]=\langle\phi, \psi\rangle
$$

for all $\phi, \psi \in C^{\infty}\left(\mathbb{T}^{2}\right)$ (Gaussian means that the real-valued r.v. $\langle\omega, \phi\rangle$ is Gaussian, for every $\left.\phi \in C^{\infty}\left(\mathbb{T}^{2}\right)\right)$. We have denoted by $\langle\omega(\theta), \phi\rangle$ the duality between the distribution $\omega(\theta)$ (for some $\theta \in \Xi$ ) and the test function $\phi \in C^{\infty}\left(\mathbb{T}^{2}\right)$. These properties uniquely characterize the law of $\omega$. In more heuristic terms, as it is often written in the physics literature,

$$
\mathbb{E}[\omega(x) \omega(y)]=\delta(x-y)
$$

since double integration of this identity against $\phi(x) \psi(y)$ gives (2). White noise exists: it is sufficient to take the complete orthonormal system $\left\{e_{n}\right\}_{n \in \mathbb{Z}^{2}}$ of $L^{2}\left(\mathbb{T}^{2}, \mathbb{C}\right)$ as above, a probability space $(\Xi, \mathcal{F}, \mathbb{P})$ supporting a sequence of independent standard Gaussian variables $\left\{G_{n}\right\}_{n \in \mathbb{Z}^{2}}$, and consider the series

$$
\omega=\sqrt{2} \operatorname{Re} \sum_{n \in \mathbb{Z}^{2}} G_{n} e_{n} .
$$

The partial sums $\omega_{N}^{\mathbb{C}}(\theta, x)=\sum_{|n| \leq N} G_{n}(\theta) e_{n}(x)$ are well defined complex valued random fields with square integrable paths, $\omega_{N}: \Xi \rightarrow L^{2}\left(\mathbb{T}^{2}, \mathbb{C}\right)$. For every $\epsilon>0,\left\{\omega_{N}^{\mathbb{C}}\right\}_{N \in \mathbb{N}}$ is a Cauchy sequence in $L^{2}\left(\Xi ; H^{-1-\epsilon}\left(\mathbb{T}^{2}, \mathbb{C}\right)\right)$, because

$$
\begin{aligned}
\mathbb{E}\left[\left\|\omega_{N}^{\mathbb{C}}(\theta, x)-\omega_{M}^{\mathbb{C}}(\theta, x)\right\|_{H^{-1-\epsilon}}^{2}\right]=\mathbb{E}\left[\sum_{M<|n| \leq N}\left(1+|n|^{2}\right)^{-1-\epsilon}\left|G_{n}\right|^{2}\right] \\
=\sum_{M<|n| \leq N}\left(1+|n|^{2}\right)^{-1-\epsilon} .
\end{aligned}
$$

The limit $\omega^{\mathbb{C}}$ in $L^{2}\left(\Xi ; H^{-1-\epsilon}\left(\mathbb{T}^{2}, \mathbb{C}\right)\right)$ thus exists, and $\omega=\sqrt{2} \operatorname{Re} \omega^{\mathbb{C}}$ is a white noise because (doing rigorously the computation on the partial sums and then taking the limit) it is centered and for $\phi, \psi \in C^{\infty}\left(\mathbb{T}^{2}\right)$,

$$
\begin{aligned}
\mathbb{E}[\langle\omega, \phi\rangle\langle\omega, \psi\rangle] & =\operatorname{Re} \mathbb{E}\left[\left\langle\omega_{C}, \phi\right\rangle\left\langle\overline{\omega_{C}}, \psi\right\rangle\right]=\operatorname{Re} \sum_{n, m \in \mathbb{Z}^{2}}\left\langle e_{n}, \phi\right\rangle \overline{\left\langle e_{m}, \psi\right\rangle} \mathbb{E}\left[G_{n} G_{m}\right] \\
& =\operatorname{Re} \sum_{n \in \mathbb{Z}^{2}}\left\langle e_{n}, \phi\right\rangle \overline{\left\langle e_{n}, \psi\right\rangle}=\langle\phi, \psi\rangle .
\end{aligned}
$$


[One obtains the same result by taking $\omega=\sum_{n \in \mathbb{Z}^{2}} G_{n} e_{n}$ where $\mathbb{Z}^{2} \backslash\{0\}$ is partitioned as $\mathbb{Z}^{2}=\Lambda \cup(-\Lambda) ; G_{n}$ are i.i.d. $N(0,1)$ on $\Lambda \cup\{0\}$ and $G_{-n}=\overline{G_{n}}$ for $n \in \Lambda$.] The law $\mu$ of the measurable map $\omega: \Xi \rightarrow H^{-1-\epsilon}\left(\mathbb{T}^{2}\right)$ is a Gaussian measure (it is sufficient to check that $\langle\omega, \phi\rangle$ is Gaussian for every $\phi \in C^{\infty}\left(\mathbb{T}^{2}\right)$, and this is true since $\langle\omega, \phi\rangle$ is the $L^{2}(\Xi)$-limit of the Gaussian variables $\left.\sum_{|n| \leq N} G_{n}\left\langle e_{n}, \phi\right\rangle\right)$. The measure $\mu$ is supported by $H^{-1-}\left(\mathbb{T}^{2}\right)$ but not by $H^{-1}\left(\mathbb{T}^{2}\right)$; namely, we have

$$
\mu\left(H^{-1}\left(\mathbb{T}^{2}\right)\right)=0 .
$$

This follows from

$$
\mathbb{E}\left[\left\|\omega^{\mathbb{C}}\right\|_{H^{-1}}^{2}\right]=\sum_{n \in \mathbb{Z}^{2}}\left(1+|n|^{2}\right)^{-1}=+\infty .
$$

The measure $\mu$ is sometimes denoted heuristically as

$$
\mu(d \omega)=\frac{1}{Z} \exp \left(-\frac{1}{2} \int_{\mathbb{T}^{2}} \omega^{2} d x\right) d \omega
$$

and called the enstrophy measure.

The notation " $d \omega "$ has no meaning (unless interpreted as a limit of measures on finite dimensional Euclidean spaces), it just reminds the structure of centered nonsingular Gaussian measures in $\mathbb{R}^{n}$, that is $\mu_{n}\left(d \omega_{n}\right)=\frac{1}{Z_{n}} \exp \left(-\frac{1}{2}\left\langle Q_{n}^{-1} \omega_{n}, \omega_{n}\right\rangle_{\mathbb{R}^{n}}\right) d \omega_{n}$ where $d \omega_{n}$ is Lebesgue measure in $\mathbb{R}^{n}$ and $Q_{n}$ is the covariance matrix. The notation $\int_{\mathbb{T}^{2}} \omega^{2} d x$ alludes to the fact that $\mu$, heuristically considered as a Gaussian measure on $L^{2}\left(\mathbb{T}^{2}\right)$ (this is not possible, $\left.\mu\left(L^{2}\left(\mathbb{T}^{2}\right)\right)=0\right)$, has covariance equal to the identity: if $Q=I d$, then $\left\langle Q^{-1} \omega, \omega\right\rangle_{L^{2}}=\int_{\mathbb{T}^{2}} \omega^{2} d x$. The fact that in $L^{2}\left(\mathbb{T}^{2}\right)$ the covariance operator $Q$, heuristically defined as

$$
\langle Q \omega, \omega\rangle_{L^{2}}=\mathbb{E}\left[\langle\omega, \phi\rangle_{L^{2}}\langle\omega, \psi\rangle_{L^{2}}\right]
$$

is the identity in the case of the law $\mu$ of white noise, is a simple "consequence" (the argument is not rigorous ab initio) of the definition (2) of white noise.

\subsection{Weak formulation of mSQG}

Before we can turn to the construction of a white noise solution, we need to fix our notion of solution in such a weak setting; especially, we need to find a good interpretation of the nonlinear term. To this end, we integrate (10) in time. Using that $u$ is divergence free, we get for any test-function $\phi \in C^{\infty}\left(\mathbb{T}^{2}\right)$

$$
\left\langle\theta_{t}, \phi\right\rangle=\left\langle\theta_{0}, \phi\right\rangle+\int_{0}^{t}\left\langle\theta_{s} u_{s}, \nabla \phi\right\rangle \mathrm{d} s
$$

From $u=\nabla^{\perp} \Lambda^{-1-\epsilon} \theta$, it follows

$$
u_{t}(x)=\int_{\mathbb{T}^{2}} K(x-y) \theta_{t}(y) \mathrm{d} y
$$


where the kernel $K$ is in the whole space given by $K(x)=c \frac{x^{\perp}}{|x|^{3-\epsilon}}$ for some constant $c>0$. In the torus, we have $K$ smooth for $x \neq 0, K(x-y)=-K(y-x)$ and $K(x) \leq \frac{c}{|x|^{2-\epsilon}}$ for $|x|$ small. We set $K(0)=0$. By the symmetry of the kernel, we obtain the Schochet symmetrisation

$$
\begin{aligned}
\int_{0}^{t}\left\langle\theta_{s} u_{s}, \nabla \phi\right\rangle \mathrm{d} s & =\int_{0}^{t} \int_{\mathbb{T}^{2}} \int_{\mathbb{T}^{2}} \theta_{s}(x) K(x-y) \theta_{s}(y) \nabla \phi(x) \mathrm{d} y \mathrm{~d} x \mathrm{~d} s \\
& =\frac{1}{2} \int_{0}^{t} \int_{\mathbb{T}^{2}} \int_{\mathbb{T}^{2}} \theta_{s}(x) K(x-y) \theta_{s}(y) \nabla(\phi(x)-\phi(y)) \mathrm{d} y \mathrm{~d} x \mathrm{~d} s \\
& =\frac{1}{2} \int_{0}^{t} \int_{\mathbb{T}^{2}} \int_{\mathbb{T}^{2}} \theta_{s}(x) \theta_{s}(y) H_{\phi}(x, y) \mathrm{d} y \mathrm{~d} x \mathrm{~d} s
\end{aligned}
$$

where we set $H_{\phi}(x, y)=K(x-y) \nabla(\phi(x)-\phi(y))$. Such a symmetrisation was carried out in 32 for the 2D Euler equations and it is in the same spirit as rewriting the nonlinearity with the commutator used for example in [3, 25] and [30. Due to the crucial role of $H_{\phi}$ throughout this work let us give a rigorous definition and collect some properties of it.

Definition 3 Let $K: \mathbb{T}^{2} \rightarrow \mathbb{R}^{2}$ be smooth for $x \neq 0, K(x)=-K(-x)$ for all $x \in \mathbb{T}^{2}$, $K(0)=0, K(x) \leq \frac{c}{|x|^{2-\epsilon}}$ for $|x|$ small and $\phi \in C^{\infty}\left(\mathbb{T}^{2}\right)$. Then the function

$$
H_{\phi}: \mathbb{T}^{2} \times \mathbb{T}^{2} \rightarrow \mathbb{R}, H_{\phi}(x, y)=K(x-y) \nabla(\phi(x)-\phi(y))
$$

is in $L^{2}\left(\mathbb{T}^{2} \times \mathbb{T}^{2}\right), H_{\phi}(y, x)=H_{\phi}(x, y)$ for all $x, y \in \mathbb{T}^{2}, H_{\phi}(x, x)=0$ and $H_{\phi}$ is smooth outside of the diagonal.

Our next purpose is to define

$$
\int_{\mathbb{T}^{2}} \int_{\mathbb{T}^{2}} \omega(x) \omega(y) H_{\phi}(x, y) \mathrm{d} y \mathrm{~d} x \mathrm{~d} s
$$

when $\omega: \Xi \rightarrow C^{\infty}\left(\mathbb{T}^{2}\right)^{\prime}$ is a white noise.

If $\omega \in C^{\infty}\left(\mathbb{T}^{2}\right)^{\prime}$ is a distribution, we can define a distribution $\omega \otimes \omega \in C^{\infty}\left(\mathbb{T}^{2} \times \mathbb{T}^{2}\right)^{\prime}$ by setting

$$
\langle\omega \otimes \omega, \phi \otimes \psi\rangle=\langle\omega, \phi\rangle\langle\omega, \psi\rangle \quad\left(\phi, \psi \in C^{\infty}\left(\mathbb{T}^{2}\right)\right)
$$

where $(\phi \otimes \psi)(x, y)=\phi(x) \psi(y)$ and extend this to all $f \in C^{\infty}\left(\mathbb{T}^{2} \times \mathbb{T}^{2}\right)$ by density arguments. If $\omega \in H^{-s}\left(\mathbb{T}^{2}\right)$ for some $s>0$, then $\omega \otimes \omega \in H^{-2 s}\left(\mathbb{T}^{2} \times \mathbb{T}^{2}\right)$. In the case of $\omega$ being a white noise, we have the following result for this distribution (taken from [15]).

Corollary 4 Let $\omega: \Xi \rightarrow C^{\infty}\left(\mathbb{T}^{2}\right)^{\prime}$ be a white noise and $f \in H^{2+}\left(\mathbb{T}^{2} \times \mathbb{T}^{2}\right)$.

i) We have $\mathbb{E}[\langle\omega \otimes \omega, f\rangle]=\int_{\mathbb{T}^{2}} f(x, x) \mathrm{d} x$. 
ii) If $f$ is symmetric, we have

$$
\mathbb{E}\left[|\langle\omega \otimes \omega, f\rangle-\mathbb{E}[\langle\omega \otimes \omega, f\rangle]|^{2}\right]=\int_{\mathbb{T}^{2}} \int_{\mathbb{T}^{2}} f^{2}(x, y) \mathrm{d} x \mathrm{~d} y
$$

Although our function $H_{\phi}$ is not of class $H^{2+}\left(\mathbb{T}^{2} \times \mathbb{T}^{2}\right)$, we can use this corollary to give a meaning to $\left\langle\omega \otimes \omega, H_{\phi}\right\rangle$ in $L^{2}(\Xi)$ by a suitable approximation. An obvious consequence of ii) is the following.

Corollary 5 Let $\omega: \Xi \rightarrow C^{\infty}\left(\mathbb{T}^{2}\right)^{\prime}$ be a white noise and $\phi \in C^{\infty}\left(\mathbb{T}^{2}\right)$ be given. Furthermore, let $\left(H_{\phi}^{n}\right)_{n} \in H^{2+}\left(\mathbb{T}^{2} \times \mathbb{T}^{2}\right)$ be a sequence of symmetric functions such that

$$
\left\|H_{\phi}-H_{\phi}^{n}\right\|_{L^{2}\left(\mathbb{T}^{2} \times \mathbb{T}^{2}\right)} \rightarrow 0 \quad(n \rightarrow \infty)
$$

Then $\left\langle\omega \otimes \omega, H_{\phi}^{n}\right\rangle-\mathbb{E}\left[\omega \otimes \omega, H_{\phi}^{n}\right]$ is a Cauchy sequence in $L^{2}(\Xi)$.

Choosing the sequence $H_{\phi}^{n}$ a bit more specific, we obtain the following convergence result. More precisely, we take a sequence such that $\mathbb{E}\left[\omega \otimes \omega, H_{\phi}^{n}\right]$ vanishes in the limit, and thus, we somehow normalize our limit to have expectation 0.

Corollary 6 Let $\omega, \phi$ and $\left(H_{\phi}^{n}\right)_{n}$ be as above and assume additionally

$$
\int_{\mathbb{T}^{2}} H_{\phi}^{n}(x, x) \mathrm{d} x \rightarrow 0 \quad(n \rightarrow \infty) .
$$

Then, $\left\langle\omega \otimes \omega, H_{\phi}^{n}\right\rangle$ is a Cauchy sequence in $L^{2}(\Xi)$ and we denote its limit by $\left\langle\omega \otimes \omega, H_{\phi}\right\rangle$. This limit does not depend on the specific choice of $\left(H_{\phi}^{n}\right)_{n}$, and we have $\mathbb{E}\left[\left|\left\langle\omega \otimes \omega, H_{\phi}\right\rangle\right|^{2}\right]=$ $\left\|H_{\phi}\right\|_{L^{2}\left(\mathbb{T}^{2} \times \mathbb{T}^{2}\right)}$.

Proof. By the above corollary, we have that $\left\langle\omega \otimes \omega, H_{\phi}^{n}\right\rangle-\mathbb{E}\left[\left\langle\omega \otimes \omega, H_{\phi}^{n}\right\rangle\right]$ converges in $L^{2}(\Xi)$, and by our assumption, $\mathbb{E}\left[\omega \otimes \omega, H_{\phi}^{n}\right]=\int_{\mathbb{T}^{2}} H_{\phi}^{n}(x, x) \mathrm{d} x \rightarrow 0 \quad(n \rightarrow \infty)$, so $\left\langle\omega \otimes \omega, H_{\phi}^{n}\right\rangle$ converges in $L^{2}(\Xi)$. For the estimate, note that

$$
\begin{aligned}
\mathbb{E}\left[\left|\left\langle\omega \otimes \omega, H_{\phi}\right\rangle\right|^{2}\right] & =\lim _{n \rightarrow \infty} \mathbb{E}\left[\left|\left\langle\omega \otimes \omega, H_{\phi}^{n}\right\rangle\right|^{2}\right] \\
& =\lim _{n \rightarrow \infty} \mathbb{E}\left[\left|\left\langle\omega \otimes \omega, H_{\phi}^{n}\right\rangle-\mathbb{E}\left[\left\langle\omega \otimes \omega, H_{\phi}^{n}\right\rangle\right]\right|^{2}\right] \\
& =\lim _{n \rightarrow \infty}\left\|H_{\phi}^{n}\right\|_{L^{2}\left(\mathbb{T}^{2} \times \mathbb{T}^{2}\right)}^{2}=\left\|H_{\phi}\right\|_{L^{2}\left(\mathbb{T}^{2} \times \mathbb{T}^{2}\right)}^{2} .
\end{aligned}
$$


Remark 7 Constructing such a sequence is straight forward by cutting out the diagonal with some smooth cut-off function: Let $\varphi$ be smooth, symmetric, with $\operatorname{supp} \varphi \in B(0,1)$. $0 \leq \varphi \leq 1, \varphi \equiv 1$ in $B(0,1 / 2)$. We define $\varphi_{n}:=\varphi(n x)$ and set

$$
H_{\phi}^{n}(x, y):=\left(1-\varphi_{n}(x-y)\right) H_{\phi}(x, y) .
$$

By definition we have $H_{\phi}^{n}(x, x)=0$ and

$$
\begin{aligned}
\left\|H_{\phi}-H_{\phi}^{n}\right\|_{L^{2}\left(\mathbb{T}^{2} \times \mathbb{T}^{2}\right)}^{2} & =\int_{\mathbb{T}^{2}} \int_{\mathbb{T}^{2}}\left|H_{\phi}(x, y)\right|^{2} \varphi_{n}^{2}(x-y) \mathrm{d} x \mathrm{~d} y \leq \int_{\mathbb{T}^{2}} \int_{|x-y| \leq \frac{1}{n}}\left|H_{\phi}(x, y)\right|^{2} \mathrm{~d} x \mathrm{~d} y \\
& \leq \int_{\mathbb{T}^{2}} \int_{|x-y| \leq \frac{1}{n}}|x-y|^{-2+2 \epsilon} \mathrm{d} x \mathrm{~d} y \\
& \leq c \frac{1}{n^{2 \epsilon}}
\end{aligned}
$$

so $H_{\phi}^{n}$ has the desired properties to apply the previous corollary.

For $\epsilon=0$, we have that $H_{\phi} \notin L^{2}\left(\mathbb{T}^{2} \times \mathbb{T}^{2}\right)$ but only $H_{\phi} \in L^{p}\left(\mathbb{T}^{2} \times \mathbb{T}^{2}\right)$ for $1 \leq p<2$, and thus, the construction here does not work $\left(\left\|H_{\phi}-H_{\phi}^{n}\right\|_{L^{2}\left(\mathbb{T}^{2} \times \mathbb{T}^{2}\right)}\right.$ diverges logarithmically).

The above results also hold when we have an additional time dependence, and thus, they give rise to a good notion of a weak solution.

Theorem 8 Let $\theta: \Xi \times[0, T] \rightarrow C^{\infty}\left(\mathbb{T}^{2}\right)^{\prime}$ be measurable with $\theta(\xi) \in C\left([0, T] ; H^{-1-}\left(\mathbb{T}^{2}\right)\right)$. Assume that $\theta_{t}$ is a white noise for all $t \in[0, T]$. For $\phi \in C^{\infty}\left(\mathbb{T}^{2}\right)$ let $H_{\phi}^{n}$ be an approximation of $H_{\phi}$ as in Corollary 6 . Then, $\left\langle\theta . \otimes \theta\right.$. $\left.H_{\phi}^{n}\right\rangle$ is a Cauchy sequence in $L^{2}\left(\Xi ; L^{2}(0, T)\right)$ and we denote the limit by $\left\langle\theta . \otimes \theta ., H_{\phi}\right\rangle$.

We now have everything in place to define what we mean by a white noise solution of mSQG.

Definition 9 Let $\theta: \Xi \times[0, T] \rightarrow C^{\infty}\left(\mathbb{T}^{2}\right)^{\prime}$ be measurable with $\theta(\xi) \in C\left([0, T] ; H^{-1-}\left(\mathbb{T}^{2}\right)\right)$ for almost all $\xi \in \Xi$. We call $\theta$ a white noise solution of $m S Q G$ if $\theta_{t}$ is a white noise for all $t \in[0, T]$ and

$$
\left\langle\theta_{t}, \phi\right\rangle=\left\langle\theta_{0}, \phi\right\rangle+\int_{0}^{t}\left\langle\theta_{s} \otimes \theta_{s}, H_{\phi}\right\rangle \mathrm{d} s
$$

P-a.s. for every $\phi \in C^{\infty}\left(\mathbb{T}^{2}\right)$. 


\section{Random point vortex dynamics}

The first step to construct a white noise solution is to consider a finite number of particles whose interaction is described by the kernel $K$. We define

$$
\Delta_{N}:=\left\{\left(x_{1}, \ldots, x_{N}\right) \in\left(\mathbb{T}^{2}\right)^{N}: x_{i}=x_{j} \text { for some } i \neq j, i, j=1, \ldots, N\right\} .
$$

The dynamics of point vortices are given by the finite dimensional system

$$
\frac{d x_{i}(t)}{d t}=\frac{1}{\sqrt{N}} \sum_{j=1}^{N} \xi_{j} K\left(x_{i}(t)-x_{j}(t)\right), \quad i=1, \ldots, N
$$

where $\xi_{j} \in \mathbb{R}$ is the intensity of the vortex $x_{j}(t)$ and the initial value $\left(x_{1}(0), \ldots, x_{N}(0)\right) \in$ $\Delta_{N}^{c}$ is given. Due to $K(0)=0$ the term with $i=j$ in the sum vanishes. First we show that for a.e. $\left(x_{1}(0), \ldots, x_{N}(0)\right) \in \Delta_{N}^{c}$ the point vortex dynamics are globally well-defined, where we consider the Lebesgue measure on the torus. This is obviously the case if there is no collision, i.e., if $x(t):=\left(x_{1}(t), \ldots, x_{N}(t) \in \Delta_{N}^{c}\right)$ for all $t$.

\subsection{No collision for a.e. initial condition}

We present a variant of a famous result of solvability for a.e. initial conditions, taken from [14] (on the torus) and [26], Chapter 4 (in full space). We work on the unitary torus $\mathbb{T}^{2}=\mathbb{R}^{2} / \mathbb{Z}^{2}$, as in [14]; here Lebesgue measure is a probability, and particle displacement is controlled a priori by the compactness of the set. Recall that the singular part of interaction kernel $K(x-y)$ on $\mathbb{T}^{2}$ is locally of the form

$$
K(x-y) \sim \frac{(x-y)^{\perp}}{|x-y|^{3-\epsilon}}
$$

for some $\epsilon>0$. The function $K$ is given by the orthogonal gradient of a certain Green-type function $G$ :

$$
K(x)=\nabla^{\perp} G(x)
$$

and the singular part of $G(x)$ is locally of the form (up to multiplicative constants)

$$
G(x-y) \sim \frac{1}{|x-y|^{1-\epsilon}} .
$$

We introduce the (Lyapunov) function

$$
L\left(x_{1}, \ldots, x_{N}\right)=-\sum_{\substack{i, j=1, \ldots, N \\ i \neq j}} G\left(x_{i}-x_{j}\right) .
$$


By a simple argument, if we prove for a given an initial condition

$$
L\left(x_{1}(t), \ldots, x_{N}(t)\right) \leq C
$$

then particles do not collapse and the dynamics is globally defined for that initial condition, because $G$ is strictly negative in a neighborhood of the singularity.

With $L(t)=L\left(x_{1}(t), \ldots, x_{N}(t)\right)$, we have

$$
\begin{aligned}
\frac{d L(t)}{d t}=-\sum_{\substack{i, j=1, \ldots, N \\
i \neq j}} \nabla G\left(x_{i}(t)-x_{j}(t)\right)\left(\frac{d x_{i}(t)}{d t}-\frac{d x_{j}(t)}{d t}\right) \\
=-\sum_{\substack{i, j=1, \ldots, N \\
i \neq j}} \nabla G\left(x_{i}(t)-x_{j}(t)\right) \\
\quad \frac{1}{\sqrt{N}}\left(\sum_{k \neq i} \xi_{k} \nabla^{\perp} G\left(x_{i}(t)-x_{k}(t)\right)-\sum_{k \neq j} \xi_{k} \nabla^{\perp} G\left(x_{j}(t)-x_{k}(t)\right)\right) .
\end{aligned}
$$

Here we see an important cancellation (its importance will be appreciated below): the term in the sum $\sum_{k \neq i}$ with $k=j$ and the term in the sum $\sum_{k \neq j}$ with $k=i$ do not contribute, because

$$
\nabla G\left(x_{i}(t)-x_{j}(t)\right) \cdot \nabla^{\perp} G\left(x_{i}(t)-x_{j}(t)\right)=0 .
$$

These are the most singular terms, since for small $\left|x_{i}(t)-x_{j}(t)\right|$ they behave like

$$
\frac{1}{\left|x_{i}(t)-x_{j}(t)\right|^{4-2 \epsilon}} \text {. }
$$

The other terms behave like

$$
\frac{1}{\left|x_{i}(t)-x_{j}(t)\right|^{2-\epsilon}} \frac{1}{\left|x_{i}(t)-x_{k}(t)\right|^{2-\epsilon}}
$$

with $j \neq k$; hence, they are less singular when two particles approach each other.

In order to make progresses, we now need to consider the flow map $x^{0} \mapsto x\left(t \mid x^{0}\right)$. This is locally defined, when $x^{0} \in \Delta_{N}^{c}$. However, the time before collision depends on $x^{0}$ and complicate matters. To avoid these troubles, we mollify $G$ in such a way that we have global solutions for all $x^{0}$, a smooth flow, but also equal to the original solutions if particles are not too close each other.

For every $\delta \in(0,1)$, denote by $G^{(\delta)}(x)$ a smooth periodic function such that

$$
\begin{gathered}
G^{(\delta)}(x)=G(x) \text { for }|x| \geq \delta \\
\left|G^{(\delta)}(x)\right| \leq \frac{C}{|x-y|^{1-\epsilon}} \text { for }|x|>0 \\
\left|\nabla G^{(\delta)}(x)\right| \leq \frac{C}{|x-y|^{2-\epsilon}} \text { for }|x|>0 .
\end{gathered}
$$


Denote by $\left(x_{1}^{(\delta)}(t), \ldots, x_{N}^{(\delta)}(t)\right)$ the unique solution of

$$
\frac{d x_{i}^{(\delta)}(t)}{d t}=\frac{1}{\sqrt{N}} \sum_{j \neq i} \xi_{j} K^{(\delta)}\left(x_{i}^{(\delta)}(t)-x_{j}^{(\delta)}(t)\right), \quad i=1, \ldots, N
$$

with given (arbitrary) initial condition.

Lemma 10 Consider the smooth map $x^{0} \mapsto x_{i}^{(\delta)}\left(t \mid x^{0}\right)$ in $\left(\mathbb{T}^{2}\right)^{N}$. Then, the probability product measure Leb $2 N$ on $\left(\mathbb{T}^{2}\right)^{N}$ is invariant for this map.

Proof. It is a known fact for smooth flows that the determinant is given by the exponential of the divergence of the vector field, which here is zero; hence, the determinant is identically equal to one and the flow is Lebesgue measure preserving. Let us only check that the divergence is zero: it is the sum of divergences on each component $\mathbb{T}^{2}$ of $\left(\mathbb{T}^{2}\right)^{N}$, which are all equal to zero because the components have the form $\nabla^{\perp} \varphi^{(\delta)}(x)$ (apply Schwarz theorem on mixed second derivatives).

Similarly to above, let us introduce the function

$$
L^{(\delta)}\left(x_{1}, \ldots, x_{N}\right)=-\sum_{\substack{i, j=1, \ldots, N \\ i \neq j}}\left(G^{(\delta)}\left(x_{i}-x_{j}\right)-k\right)
$$

where $k$ is such that $-\left(G^{(\delta)}(x)-k\right) \geq 0$ for all $x \in\left(\mathbb{T}^{2}\right)^{N}$.

Setting $L^{(\delta)}(t)=L^{(\delta)}\left(x_{1}^{(\delta)}(t), \ldots, x_{N}^{(\delta)}(t)\right)$, we have

$$
\begin{aligned}
\frac{d L^{(\delta)}(t)}{d t} & =-\sum_{\substack{i, j=1, \ldots, N \\
i \neq j}} \nabla G^{(\delta)}\left(x_{i}^{(\delta)}(t)-x_{j}^{(\delta)}(t)\right) . \\
& \cdot \frac{1}{\sqrt{N}}\left(\sum_{k \neq i} \xi_{k} \nabla^{\perp} G^{(\delta)}\left(x_{i}^{(\delta)}(t)-x_{k}^{(\delta)}(t)\right)-\sum_{k \neq j} \xi_{k} \nabla^{\perp} G^{(\delta)}\left(x_{j}^{(\delta)}(t)-x_{k}^{(\delta)}(t)\right)\right) .
\end{aligned}
$$

Again the terms in the last two sums of the form $\nabla^{\perp} G^{(\delta)}\left(x_{i}^{(\delta)}(t)-x_{j}^{(\delta)}(t)\right)$ cancel with $\nabla G^{(\delta)}\left(x_{i}^{(\delta)}(t)-x_{j}^{(\delta)}(t)\right)$. Using this estimate and the invariance of Lebesgue measure we can prove:

Lemma 11 There exists a constant $C>0$ such that, for all $\delta \in(0,1)$,

$$
-\int_{\left(\mathbb{T}^{2}\right)^{N}} \sup _{t \in[0, T]} \sum_{\substack{i, j=1, \ldots, N \\ i \neq j}}\left(G^{(\delta)}\left(x_{i}^{(\delta)}\left(t \mid x^{0}\right)-x_{j}^{(\delta)}\left(t \mid x^{0}\right)\right)-k\right) d x^{0} \leq C .
$$


Proof. We may summarize the last identity above in the form

$$
\begin{aligned}
& \frac{d L^{(\delta)}(t)}{d t}=\frac{1}{\sqrt{N}} \sum_{\substack{i, j, k=1, \ldots, N \\
i \neq j, i \neq k, j \neq k}} a_{i j k} J_{i, j, k}^{(\delta)}\left(x^{(\delta)}(t)\right) \\
& J_{i, j, k}^{(\delta)}(x):=\nabla G^{(\delta)}\left(x_{i}-x_{j}\right) \cdot \nabla^{\perp} G^{(\delta)}\left(x_{i}-x_{k}\right)
\end{aligned}
$$

for suitable coefficients $a_{i j k}$. Integrating in time, taking the supremum in time over $[0, T]$ and then integrating with respect to the initial condition $x^{0}$, we have

$$
\begin{aligned}
\int_{\left(\mathbb{T}^{2}\right)^{N}} \sup _{t \in[0, T]} L^{(\delta)}(t) d x^{0} \leq & \int_{\left(\mathbb{T}^{2}\right)^{N}}\left|L^{(\delta)}(0)\right| d x^{0} \\
& +\frac{C}{\sqrt{N}} \sum_{\substack{i, j, k=1, \ldots, N \\
i \neq j, i \neq k, j \neq k}} \int_{0}^{T}\left(\int_{\left(\mathbb{T}^{2}\right)^{N}}\left|J_{i, j, k}^{(\delta)}\left(x^{(\delta)}\left(s \mid x^{0}\right)\right)\right| d x^{0}\right) d s .
\end{aligned}
$$

Now we use the most essential ingredient: the invariance of Lebesgue measure under the map $x^{0} \mapsto x^{(\delta)}\left(s \mid x^{0}\right)$. This gives us

$$
\int_{\left(\mathbb{T}^{2}\right)^{N}}\left|J_{i, j, k}^{(\delta)}\left(x^{(\delta)}\left(s \mid x^{0}\right)\right)\right| d x^{0}=\int_{\left(\mathbb{T}^{2}\right)^{N}}\left|J_{i, j, k}^{(\delta)}\left(x^{0}\right)\right| d x^{0} .
$$

From the properties imposed on $G^{(\delta)}$ we have

$$
\left|J_{i, j, k}^{(\delta)}\left(x^{0}\right)\right| \leq \frac{C}{\left|x_{i}-x_{j}\right|^{2-\epsilon}\left|x_{i}-x_{k}\right|^{2-\epsilon}}
$$

for some constant $C>0$; hence,

$$
\int_{\left(\mathbb{T}^{2}\right)^{N}}\left|J_{i, j, k}^{(\delta)}\left(x^{0}\right)\right| d x^{0} \leq C \int_{\left(\mathbb{T}^{2}\right)^{2}} \frac{1}{\left|x_{i}-x_{k}\right|^{2-\epsilon}}\left(\int_{\mathbb{T}^{2}} \frac{1}{\left|x_{i}-x_{j}\right|^{2-\epsilon}} d x_{j}\right) d x_{i} d x_{k} \leq C^{\prime}
$$

for some constant $C^{\prime}>0$. Similarly, $\int_{\left(\mathbb{T}^{2}\right)^{N}}\left|L^{(\delta)}(0)\right| d x^{0} \leq C^{\prime \prime}$ for some constant $C^{\prime}>0$. In conclusion,

$$
-\int_{\left(\mathbb{T}^{2}\right)^{N}} \sup _{t \in[0, T]} \sum_{\substack{i, j=1, \ldots, N \\ i \neq j}}\left(G^{(\delta)}\left(x_{i}^{(\delta)}\left(t \mid x^{0}\right)-x_{j}^{(\delta)}\left(t \mid x^{0}\right)\right)-k\right) d x^{0} \leq C
$$

for some constant $C>0$.

Remark 12 Without the cancellation of the most singular terms, we would have also

$$
\int_{\left(\mathbb{T}^{2}\right)^{2}} \frac{1}{\left|x_{i}-x_{j}\right|^{4-4 \epsilon}} d x_{i} d x_{j}=+\infty
$$

(for $\epsilon$ small). 
Theorem 13 For Lebesgue a.e. initial condition $x^{0} \in \Delta_{N}^{c}$, there is no collision and the solution is global and unique.

Proof. Denote by $d_{T}^{(\delta)}\left(x^{0}\right)$ the minimal distance between vortices of the smoothed system, starting from $x^{0}$, over $[0, T]$. Then

$$
\begin{aligned}
& d_{T}^{(\delta)}\left(x^{0}\right)<\delta \Longleftrightarrow \Longleftrightarrow t \in[0, T], \exists i \neq j:\left|x_{i}^{(\delta)}\left(t \mid x^{0}\right)-x_{j}^{(\delta)}\left(t \mid x^{0}\right)\right|<\delta \\
& \Longrightarrow-\sup _{t \in[0, T]} \sum_{\substack{i, j=1, \ldots ., N \\
i \neq j}}\left(G^{(\delta)}\left(x_{i}^{(\delta)}\left(t \mid x^{0}\right)-x_{j}^{(\delta)}\left(t \mid x^{0}\right)\right)-k\right) \\
&>C\left(1+\frac{1}{\delta^{1-\epsilon}}\right)
\end{aligned}
$$

hence

$$
\begin{aligned}
& \operatorname{Leb}_{2 N}\left\{x^{0} \in\left(\mathbb{T}^{2}\right)^{N}: d_{T}^{(\delta)}\left(x^{0}\right)<\delta\right\} \\
& \leq \operatorname{Leb}_{2 N}\left\{x^{0} \in\left(\mathbb{T}^{2}\right)^{N}:\right. \\
& \left.\quad-\sup _{t \in[0, T]} \sum_{\substack{i, j=1, \ldots, N \\
i \neq j}}\left(G^{(\delta)}\left(x_{i}^{(\delta)}\left(t \mid x^{0}\right)-x_{j}^{(\delta)}\left(t \mid x^{0}\right)\right)-k\right)>C\left(1+\frac{1}{\delta^{1-\epsilon}}\right)\right\} \\
& \leq \frac{C}{C\left(1+\frac{1}{\delta^{1-\epsilon}}\right)}
\end{aligned}
$$

where we have used Chebyshev inequality and the lemma and have assumed $\delta$ so small that $C\left(1+\frac{1}{\delta^{1-\epsilon}}\right)>0$. Thus, for a very large (in the sense of Lebesgue measure) set of initial conditions $d_{T}^{(\delta)}\left(x^{0}\right) \geq \delta$, which means that $x^{(\delta)}\left(t \mid x^{0}\right)=x\left(t \mid x^{0}\right)$ and no collision occurs. This property is true for a.e. initial conditions, by the arbitrarity of $\delta$.

Lemma 14 The map $x^{0} \mapsto x\left(t \mid x^{0}\right)$ defined a.e. by the previous theorem is measurable and preserves Lebesgue measure.

Proof. The claim follows directly from the fact that $x\left(t \mid x^{0}\right)=x^{(\delta)}\left(t \mid x^{0}\right)$ for some sufficiently small $\delta>0$ depending on $x^{0}$ and $t$ and that the Lebesgue measure is invariant under the smooth map $x^{0} \mapsto x^{(\delta)}\left(t \mid x^{0}\right)$.

A direct consequence of the previous lemma is that if we consider the initial condition as a random variable the process defined by Theorem 13 is stationary. Altogether, we obtain the following theorem on the existence of the vortex dynamics.

Theorem 15 For every $\left(\xi_{1}, \ldots, \xi_{N}\right) \in \mathbb{R}^{N}$ and for Leb $b_{2 N}$-a.e. $X_{0}^{N} \in \Delta_{N}^{c}$, there is a unique solution $X^{N}:[0, \infty) \rightarrow \Delta_{N}^{c}$ to (3).

Considering the initial condition as a random variable distributed as Leb ${ }_{2 N}$ the stochastic process $\left(X_{t}^{N}\right)_{t}$ is stationary with invariant marginal law Leb $b_{2 N}$. 


\section{Construction of a white noise solution}

Now let us consider an i.i.d. sequence $\left(\xi_{n}\right)$ of $N(0,1)$ random variables for the intensities and an i.i.d. sequence $\left(X_{0}^{n}\right)$ of uniformly distributed $\mathbb{T}^{2}$-valued random variables for the initial positions, both on a probability space $(\Xi, \mathcal{F}, \mathbb{P})$ and independent of each other. We denote by

$$
\lambda_{N}^{0}:=\left(\otimes_{N} N(0,1)\right) \otimes L e b_{2 N}
$$

the law of the random vector $\left(\left(\xi_{1}, X_{0}^{1}\right), \ldots,\left(\xi_{N}, X_{0}^{N}\right)\right)$. Then, by Theorem 15] we have that for a.e. value of $\left(\left(\xi_{1}, X_{0}^{1}\right), \ldots,\left(\xi_{N}, X_{0}^{N}\right)\right)$ there is a unique solution $X^{N}:[0, \infty) \rightarrow \Delta_{N}^{c}$ to (3). We are interested in a measure-valued vorticity field $\theta_{N}$ which is a linear combination of the processes $X^{N}$ weighted with the intensities. For this field we obtain results similar to those for the white noise and it will be the basis to construct a white noise solution to mSQG.

Theorem 16 Let the intensities and initial positions $\left(\xi, X_{0}\right):=\left(\left(\xi_{1}, X_{0}^{1}\right), \ldots,\left(\xi_{N}, X_{0}^{N}\right)\right)$ be distributed as $\lambda_{N}^{0}$ and $\left(X_{.}^{1}, \ldots, X^{N}\right):(0, \infty) \rightarrow \Delta_{N}^{c}$ be the corresponding dynamics. Then we have for the measure-valued field

$$
\theta_{t}^{N}\left(\xi, X_{0}\right):=\frac{1}{\sqrt{N}} \sum_{n=1}^{N} \xi_{n} \delta_{X_{t}^{n}\left(\xi, X_{0}\right)}
$$

i) $\theta_{t}^{N}$ is stationary and space homogeneous.

ii) $\partial_{t}\left\langle\theta_{t}^{N}, \phi\right\rangle=\left\langle\theta_{t}^{N} \otimes \theta_{t}^{N}, H_{\phi}\right\rangle$ for all $t>0$ and $\phi \in C^{\infty}\left(\mathbb{T}^{2}\right)$.

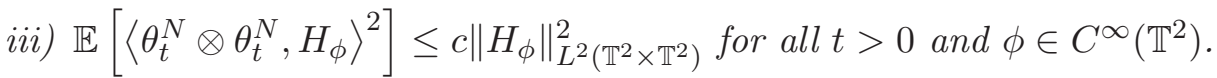

iv) $\mathbb{E}\left[\left\|\theta_{t}^{N}\right\|_{H^{-1-\delta}\left(\mathbb{T}^{2}\right)}^{p}\right] \leq c_{p, \delta}$ for all $p \geq 1, \delta>0$ independent of $t$ and $N$.

v) $\theta_{t}^{N} \stackrel{\text { law }}{\longrightarrow} \theta_{W N}$ for all $t>0$, where $\theta_{W N}$ denotes white noise and convergence takes place in $H^{-1-\delta}\left(\mathbb{T}^{2}\right)$ for all $\delta>0$.

Proof. We only show ii) and iii) here; for the other points, we refer to [15]. By definition 
of $\theta_{t}^{N}$ we have for $t>0$ and $\phi \in C^{\infty}\left(\mathbb{T}^{2}\right)$

$$
\begin{aligned}
\partial_{t}\left\langle\theta_{t}^{N}, \phi\right\rangle & =\partial_{t} \frac{1}{\sqrt{N}} \sum_{n=1}^{N} \xi_{n} \phi\left(X_{t}^{n}\right)=\frac{1}{\sqrt{N}} \sum_{n=1}^{N} \xi_{n}\left(\partial_{t} X_{t}^{n}\right) \nabla \phi\left(X_{t}^{n}\right) \\
& =\frac{1}{N} \sum_{n=1}^{N} \sum_{i=1}^{N} \xi_{n} \xi_{i} K\left(X_{t}^{n}-X_{t}^{i}\right) \nabla \phi\left(X_{t}^{n}\right) \\
& =\frac{1}{2 N} \sum_{n=1}^{N} \sum_{i=1}^{N} \xi_{n} \xi_{i} K\left(X_{t}^{n}-X_{t}^{i}\right)\left(\nabla \phi\left(X_{t}^{n}\right)-\nabla \phi\left(X_{t}^{i}\right)\right) \\
& =\frac{1}{N} \sum_{n=1}^{N} \sum_{i=1}^{N} \xi_{n} \xi_{i} H_{\phi}\left(X_{t}^{n}, X_{t}^{i}\right)=\frac{1}{N} \sum_{n=1}^{N} \sum_{i=1}^{N} \xi_{n} \xi_{i}\left\langle\delta_{X_{t}^{n}} \otimes \delta_{X_{t}^{i}}, H_{\phi}\right\rangle \\
& =\left\langle\theta_{t}^{N} \otimes \theta_{t}^{N}, H_{\phi}\right\rangle,
\end{aligned}
$$

which is the equality ii). This equality also yields

$$
\left\langle\theta_{t}^{N} \otimes \theta_{t}^{N}, H_{\phi}\right\rangle^{2}=\frac{1}{N^{2}} \sum_{n=1}^{N} \sum_{i=1}^{N} \sum_{m=1}^{N} \sum_{j=1}^{N} \xi_{n} \xi_{i} \xi_{m} \xi_{j} H_{\phi}\left(X_{t}^{n}, X_{t}^{i}\right) H_{\phi}\left(X_{t}^{m}, X_{t}^{j}\right)
$$

and taking the expectation, we deduce from the independence of the random variables

$$
\mathbb{E}\left[\left\langle\theta_{t}^{N} \otimes \theta_{t}^{N}, H_{\phi}\right\rangle^{2}\right]=\frac{1}{N^{2}} \sum_{n=1}^{N} \sum_{i=1}^{N} \sum_{m=1}^{N} \sum_{j=1}^{N} \mathbb{E}\left[\xi_{n} \xi_{i} \xi_{m} \xi_{j}\right] \mathbb{E}\left[H_{\phi}\left(X_{t}^{n}, X_{t}^{i}\right) H_{\phi}\left(X_{t}^{m}, X_{t}^{j}\right)\right] .
$$

On the one hand we have $\mathbb{E}\left[\xi_{n}\right]=0(1 \leq n \leq N)$ and the $\xi_{n}$ are independent, so only terms with $n=i$ and $m=j, n=m$ and $j=i$ or $n=j$ and $m=i$ contribute to the sum and on the other hand we have $H_{\phi}(x, x)=0$, so we can also exclude the case $n=i$ and $m=j$. Altogether,

$$
\begin{aligned}
\mathbb{E}\left[\left\langle\theta_{t}^{N} \otimes \theta_{t}^{N}, H_{\phi}\right\rangle^{2}\right]= & \frac{1}{N^{2}} \sum_{n=1}^{N} \sum_{i=1}^{N} \mathbb{E}\left[\xi_{n}^{2} \xi_{i}^{2}\right] \mathbb{E}\left[H_{\phi}\left(X_{t}^{n}, X_{t}^{i}\right)^{2}\right] \\
& +\frac{1}{N^{2}} \sum_{n=1}^{N} \sum_{i=1}^{N} \mathbb{E}\left[\xi_{n}^{2} \xi_{i}^{2}\right] \mathbb{E}\left[H_{\phi}\left(X_{t}^{n}, X_{t}^{i}\right) H_{\phi}\left(X_{t}^{i}, X_{t}^{n}\right)\right] \\
= & \frac{2}{N^{2}} \sum_{n=1}^{N} \sum_{i=1}^{N} \mathbb{E}\left[\xi_{n}^{2} \xi_{i}^{2}\right] \mathbb{E}\left[H_{\phi}\left(X_{t}^{n}, X_{t}^{i}\right)^{2}\right] \\
\leq & c \sup _{1 \leq n, i \leq N} \mathbb{E}\left[H_{\phi}\left(X_{t}^{n}, X_{t}^{i}\right)^{2}\right] \\
= & c\left\|H_{\phi}\right\|_{L^{2}\left(\mathbb{T}^{2} \times \mathbb{T}^{2}\right)}^{2}
\end{aligned}
$$


where we used the invariance of the Lebesgue measure under the process $X_{t}^{n}$ once again.

Part i) of the theorem also implies that the law of $\left(\left(\xi_{1}, X_{t}^{1}\right), \ldots,\left(\xi_{N}, X_{t}^{N}\right)\right)$ is $\lambda_{N}^{0}$ for all $t$. The process $\theta^{N}$ is not used directly to construct a white noise solution, but by Skorokhod's representation theorem we can find processes with the same law which converge to a white noise solution. To do so, we first have to prove tightness.

Lemma 17 Let $\theta^{N}$ be as in Theorem[16 and $Q^{N}$ denote its law. Then the sequence $\left(Q_{N}\right)_{N}$ is tight in $C^{1}\left([0, T] ; H^{-1-}\left(\mathbb{T}^{2}\right)\right)$ and converges weakly to some probability measure $Q$ in this space.

Proof. First we show that $\left(Q_{N}\right)_{N}$ is bounded in probability in $L^{p}\left((0, T) ; H^{-1-\delta}\left(\mathbb{T}^{2}\right)\right) \cap$ $W^{1,2}\left((0, T) ; H^{-\gamma}\left(\mathbb{T}^{2}\right)\right)$ for any $p \geq 1, \delta>0, \gamma>3$. Estimating the $L^{p}$-part is easy; we have by Theorem 16 and the stationarity of $\theta_{t}^{N}$

$$
\mathbb{E}\left[\int_{0}^{T}\left\|\theta_{t}^{N}\right\|_{H^{-1-\delta}\left(\mathbb{T}^{2}\right)}^{p} d t\right]=\int_{0}^{T} \mathbb{E}\left[\left\|\theta_{t}^{N}\right\|_{H^{-1-\delta}\left(\mathbb{T}^{2}\right)}^{p}\right] d t \leq c_{p, \delta} T .
$$

For the $W^{1,2}$-norm we replace $-1-\delta$ by $-\gamma$ and take $p=2$ which gives

$$
\mathbb{E}\left[\int_{0}^{T}\left\|\theta_{t}^{N}\right\|_{H^{-\gamma}\left(\mathbb{T}^{2}\right)}^{2} d t\right] \leq c_{2, \gamma} T
$$

To estimate the derivative in time let $\phi \in C^{\infty}\left(\mathbb{T}^{2}\right)$. We have

$$
\partial_{t}\left\langle\theta_{t}^{N}, \phi\right\rangle=\left\langle\theta_{t}^{N} \otimes \theta_{t}^{N}, H_{\phi}\right\rangle
$$

which implies by using Theorem [16] again

$$
\mathbb{E}\left[\left|\partial_{t}\left\langle\theta_{t}^{N}, \phi\right\rangle\right|^{2}\right]=\mathbb{E}\left[\left|\left\langle\theta_{t}^{N} \otimes \theta_{t}^{N}, H_{\phi}\right\rangle\right|\right] \leq C\left\|H_{\phi}\right\|_{L^{2}\left(\mathbb{T}^{2} \times \mathbb{T}^{2}\right)}^{2} .
$$

Choosing $\phi=e_{k}$ with $e_{k}(x)=e^{2 i k x}\left(k \in \mathbb{Z}^{2}, x \in \mathbb{T}^{2}\right)$, we obtain

$$
\left|H_{e_{k}}(x, y)\right| \leq c|k| \frac{\left|e_{k}(x)-e_{k}(y)\right|}{|x-y|^{2-\epsilon}} \leq c|k|^{2} \frac{1}{|x-y|^{1-\epsilon}}
$$

and thus, $\left\|H_{e_{k}}\right\|_{L^{2}\left(\mathbb{T}^{2} \times \mathbb{T}^{2}\right)} \leq c k^{2}$. This yields

$$
\begin{aligned}
\mathbb{E}\left[\int_{0}^{T}\left\|\partial_{t} \theta_{t}^{N}\right\|_{H^{-\gamma}\left(\mathbb{T}^{2}\right)}^{2} d t\right] & =\mathbb{E}\left[\int_{0}^{T} \sum_{k \in \mathbb{Z}^{2}}\left(1+|k|^{2}\right)^{-\gamma}\left|\left\langle\partial_{t} \theta_{t}^{N}, e_{k}\right\rangle\right|^{2} d t\right] \\
& \leq c T \sum_{k \in \mathbb{Z}^{2}}\left(1+|k|^{2}\right)^{-\gamma}|k|^{4}
\end{aligned}
$$


and the sum is bounded due to $\gamma>3$. So we have that $\left(Q_{N}\right)_{N}$ is bounded in probability in $L^{\infty-}\left((0, T) ; H^{-1-}\left(\mathbb{T}^{2}\right)\right) \cap W^{1,2}\left((0, T) ; H^{-\gamma}\left(\mathbb{T}^{2}\right)\right)$ and this space embeds compactly in $C\left([0, T], H^{-1-}\left(\mathbb{T}^{2}\right)\right)$ (see [15], Corollary 27). Applying now Prokhorov's theorem proves the weak convergence to some probability measure $Q$.

Finally, we have everything in place to prove Theorems 1 and 2, For the reader's convenience, let us repeat their statements at this point.

Theorem 18 For all $\epsilon \in(0,1)$, there exists a probability space $(\Xi, \mathcal{F}, P)$ and a measurable map $\theta .: \Xi \times[0, T] \rightarrow C^{\infty}\left(\mathbb{T}^{2}\right)^{\prime}(T>0$ arbitrary) such that

i) $\theta$. is a white noise solution to $m S Q G$ and $\left(\theta_{t}\right)$ is stationary.

ii) The random point vortex system defined on $(\Xi, \mathcal{F}, P)$ has a subsequence which converges $P$-a.s. to $\theta$ in $C\left([0, T] ; H^{-1-}\left(\mathbb{T}^{2}\right)\right)$.

Proof. From Lemma 17 and Skorokhods representation theorem, it follows that there is a probability space $(\bar{\Xi}, \overline{\mathcal{F}}, \bar{P})$ and $C\left([0, T] ; H^{-1-}\left(\mathbb{T}^{2}\right)\right)$-valued random variables $\bar{\theta}^{N_{k}}$ with law $Q_{N_{k}}$ converging to some $\bar{\theta}$. in $C\left([0, T] ; H^{-1-}\left(\mathbb{T}^{2}\right)\right)\left(Q_{N_{k}}\right.$ a subsequence of $\left.Q_{N}\right)$. By the method described in [15], Lemma 28 we can extend $(\bar{\Xi}, \overline{\mathcal{F}}, \bar{P})$ to some probability space $(\Xi, \mathcal{F}, P)$, define new processes $\theta^{N_{k}}$ with law $Q_{N_{k}}$ converging to a process $\theta$. in $C\left([0, T] ; H^{-1-}\left(\mathbb{T}^{2}\right)\right)$, and we have the representation

$$
\theta_{t}^{N_{k}}=\frac{1}{\sqrt{N_{k}}} \sum_{n=1}^{N_{k}} \xi_{n} \delta_{X_{t}^{n, N_{k}}},
$$

where $\left(X^{1, N_{k}}, \ldots, X^{N_{k}, N_{k}}\right)$ is the solution to (3) for a new random vector (without renaming) $\left(\left(\xi_{1}, X^{1, N_{k}}\right), \ldots,\left(\xi_{N}, X^{N_{k}, N_{k}}\right)\right)$ with law $\lambda_{N}^{0}$. What we have to show is that $\theta$. is a white noise solution in the sense of Definition 9. We know already that $\theta_{t}$ is a white noise for all $t$ and from

$$
\left\langle\theta_{t}^{N_{k}}, \phi\right\rangle-\left\langle\theta_{0}^{N_{k}}, \phi\right\rangle-\int_{0}^{t}\left\langle\theta_{s}^{N_{k}} \otimes \theta_{s}^{N_{k}}, H_{\phi}\right\rangle d s=0
$$

follows

$$
\begin{aligned}
& \mathbb{E}\left[\left|\left\langle\theta_{t}, \phi\right\rangle-\left\langle\theta_{0}, \phi\right\rangle-\int_{0}^{t}\left\langle\theta_{s} \otimes \theta_{s}, H_{\phi}\right\rangle d s\right| \wedge 1\right] \\
& \leq \mathbb{E}\left[\left|\left\langle\theta_{t}-\theta_{t}^{N_{k}}-\theta_{0}+\theta_{0}^{N_{k}}, \phi\right\rangle\right| \wedge 1\right]+\mathbb{E}\left[\int_{0}^{t}\left|\left\langle\left(\theta_{s} \otimes \theta_{s}\right)-\left(\theta_{s}^{N_{k}} \otimes \theta_{s}^{N_{k}}\right), H_{\phi}\right\rangle\right| d s \wedge 1\right] .
\end{aligned}
$$

The convergence of $\theta^{N_{k}}$ to $\theta$. in $C\left([0, T] ; H^{-1-}\left(\mathbb{T}^{2}\right)\right)$ implies

$$
\mathbb{E}\left[\left|\left\langle\theta_{t}-\theta_{t}^{N_{k}}, \phi\right\rangle-\left\langle\theta_{0}-\theta_{0}^{N_{k}}, \phi\right\rangle\right| \wedge 1\right] \rightarrow 0 \quad\left(N_{k} \rightarrow \infty\right) .
$$


For the nonlinear part, let $\left(H_{\phi}^{m}\right)_{m}$ be a smooth approximation of $H_{\phi}$ such that $H_{\phi}^{m}(x, y)=$ $H_{\phi}^{m}(y, x)$ and $H_{\phi}^{m}(x, x)=0$ for $x, y \in \mathbb{T}^{2}$. Then

$$
\begin{aligned}
\mathbb{E}\left[\left|\int_{0}^{t}\left\langle\theta_{s} \otimes \theta_{s}, H_{\phi}-H_{\phi}^{m}\right\rangle d s\right| \wedge 1\right] & \leq \int_{0}^{t} \mathbb{E}\left[\left|\left\langle\theta_{s} \otimes \theta_{s}, H_{\phi}-H_{\phi}^{m}\right\rangle d s\right|\right] \\
& \leq \int_{0}^{t} \mathbb{E}\left[\left|\left\langle\theta_{s} \otimes \theta_{s}, H_{\phi}-H_{\phi}^{m}\right\rangle d s\right|^{2}\right]^{1 / 2} \\
& \rightarrow 0 \quad(m \rightarrow \infty)
\end{aligned}
$$

by our definition of $\left\langle\theta_{s} \otimes \theta_{s}, H_{\phi}\right\rangle$ and

$$
\begin{aligned}
\mathbb{E}\left[\left|\int_{0}^{t}\left\langle\theta_{s}^{N_{k}} \otimes \theta_{s}^{N_{k}}, H_{\phi}-H_{\phi}^{m}\right\rangle d s\right| \wedge 1\right] & \leq \int_{0}^{t} \mathbb{E}\left[\left|\left\langle\theta_{s}^{N_{k}} \otimes \theta_{s}^{N_{k}}, H_{\phi}-H_{\phi}^{m}\right\rangle d s\right|^{2}\right]^{1 / 2} \\
& \leq c\left\|H_{\phi}-H_{\phi}^{m}\right\|_{L^{2}\left(\mathbb{T}^{2} \times \mathbb{T}^{2}\right)} \\
& \rightarrow 0 \quad(m \rightarrow \infty)
\end{aligned}
$$

independent of $N_{k}$. Thus,

$$
\mathbb{E}\left[\int_{0}^{t}\left|\left\langle\left(\theta_{s} \otimes \theta_{s}\right)-\left(\theta_{s}^{N_{k}} \otimes \theta_{s}^{N_{k}}\right), H_{\phi}\right\rangle\right| d s \wedge 1\right] \rightarrow 0 \quad\left(N_{k} \rightarrow \infty\right)
$$

and this gives

$$
\mathbb{E}\left[\left|\left\langle\theta_{t}, \phi\right\rangle-\left\langle\theta_{0}, \phi\right\rangle-\int_{0}^{t}\left\langle\theta_{s} \otimes \theta_{s}, H_{\phi}\right\rangle d s\right| \wedge 1\right]=0
$$

i.e., $\left\langle\theta_{t}, \phi\right\rangle-\left\langle\theta_{0}, \phi\right\rangle-\int_{0}^{t}\left\langle\theta_{s} \otimes \theta_{s}, H_{\phi}\right\rangle d s=0$ P-a.s. for all $\phi \in C^{\infty}\left(\mathbb{T}^{2}\right)$.

\section{References}

[1] S. Albeverio, A. B. Cruzeiro, Global flows with invariant (Gibbs) measures for Euler and Navier-Stokes two-dimensional fluids, Comm. Math. Phys. 129 (1990), 431-444, doi:10.1007/BF02097100.

[2] T. Buckmaster, A. Nahmod, G. Staffilani, K. Widmayer, The Surface Quasigeostrophic Equation With Random Diffusion, to appear in International Mathematics Research Notices (2018), doi:10.1093/imrn/rny261.

[3] T. Buckmaster, S. Shkoller, V. Vicol, Nonuniqueness of weak solutions to the SQG equation, arXiv:1610.00676 (2016).

[4] G. Cavallaro, R. Garra, C. Marchioro, Localization and stability of active scalar flows, Riv. Mat. Univ. Parma 4 (2013), no. 1, 175-196. 
[5] A. Castro, D. Córdoba, J. Gómez-Serrano, Global smooth solutions for the inviscid SQG equation, arXiv:1603.03325v3 (2017).

[6] D. Chae, P. Constantin, D. Córdoba, F. Gancedo, J. Wu, Generalized surface quasigeostrophic equations with singular velocities, Comm. Pure Appl. Math. 65 (2012), no. 8, 1037-1066, doi:10.1002/cpa.21390.

[7] D. Chae, P. Constantin, J. Wu, Inviscid Models Generalizing the Two-dimensional Euler and the Surface Quasi-geostrophic Equations, Riv. Math. Univ. Parma 202 (2011), 35-62, doi:10.1007/s00205-011-0411-5.

[8] P. Constantin, Analysis of Hydrodynamic Models CBMS-NSF Regional Conference Series in Applied Mathematics, SIAM-Society for Industrial and Applied Mathematics, 2017, doi:10.1137/1.9781611974805.

[9] P. Constantin, A. Majda, E. G. Tabak, Formation of strong fronts in the 2-D quasigeostrophic thermal active scalar, Nonlinearity 7 (1994), 1495-1533, doi: $10.1088 / 0951-7715 / 7 / 6 / 001$.

[10] P. Constantin, A.J. Majda, E. G. Tabak, Singular front formation in a model for quasigeostrophic flow, Phys. Fluids 6 (1994), no. 1, 9-11, doi:10.1063/1.868050.

[11] P. Constantin, H. Nguyen, Global Weak Solutions for SQG in Bounded Domains, Comm. Pure Appl. Math. 71 (2018), 2323-2333, doi:10.1002/cpa.21720.

[12] A. Córdoba, D. Córdoba, Uniqueness for SQG patch solutions, Trans. Amer. Math. Soc. Ser. B 5 (2018), 1-31, doi $10.1090 /$ btran/20.

[13] D. Córdoba, M. Fontelos, A. Mancho and J. Rodrigo, Evidence of singularities for a family of contour dynamics equations, Proc. Natl. Acad. Sci. 102 (2005), no. 17, 5949-5952, doi:10.1073/pnas.0501977102.

[14] D. Dürr, M. Pulvirenti, On the vortex flow in bounded domains, Comm. Math. Phys. 85, 265-273 (1982), doi:10.1007/BF01254459.

[15] F. Flandoli, Weak vorticity formulation of 2D Euler equations with white noise initial condition, arXiv:1707.08068 (2017), to appear on Comm. PDEs.

[16] F. Gancedo, Existence for the $\alpha$-patch model and the QG sharp front in Sobolev spaces, Adv. Math. 217 (2008), no. 6, 2569-2598, doi:10.1016/j.aim.2007.10.010.

[17] C. Geldhauser, M. Romito, Limit theorems and fluctuations for point vortices of generalized Euler equations, arXiv:1810.12706 (2018).

[18] C. Geldhauser, M. Romito, Point vortices for inviscid generalized surface quasigeostrophic models, arXiv:1812.05166 (2018). 
[19] Z. Hassainia, T. Hmidi, On the V-states for the generalized quasigeostrophicequations, Comm. Math. Phys. 337 (2015), no. 1, 321-377, doi:10.1007/s00220-015-2300-5.

[20] I.M. Held, R.T. Pierrehumbert, S.T. Garner, K.L. Swanson, Surface quasi-geostrophic dynamics, J. Fluid Mech. 282 (1995), 1-20, doi:10.1017/S0022112095000012.

[21] P. Isett, V. Vicol, Hölder Continuous Solutions of Active Scalar Equations, Ann. PDE 1 (2015), no. 1, Art. 2, 77 pp, doi 10.1007/s40818-015-0002-0.

[22] A. Kiselev, L. Ryzhik, Y. Yao, A. Zlatoš, Finite time singularity for themodified SQG patch equation, Ann. of Math. 184 (2016) no. 3, 909-948, doi:10.4007/annals.2016.184.3.7.

[23] A. Kiselev, Y. Yao, A. Zlatoš, Local regularity for the modified SQG patch equation, arXiv:1508.07611 (2015).

[24] A. J. Majda, A. L. Bertozzi, Vorticity and incompressible flow, Cambridge Univ. Press, 2002, doi:10.1017/S0022112003216578.

[25] F. Marchand, Existence and regularity of weak solutions to the quasi-geostrophic equations in the spaces $L^{p}$ or $\dot{H}^{-1 / 2}$, Commun. Math. Phys. 277 (2008), 45-67, doi:10.1007/s00220-007-0356-6.

[26] C. Marchioro, M. Pulvirenti, Mathematical theory of incompressible nonviscous fluids, volume 96 of Applied Mathematical Sciences, Springer-Verlag, New York, 1994, doi:10.1007/978-1-4612-4284-0.

[27] A. Nahmod, N. Pavlovic, G. Staffilani, N. Totz, Global flows with invariant measures for the inviscid modified SQG equation Stoch PDE: Anal Comp. 6 (2018), no. 2, 184-210, doi:10.1007/s40072-017-0106-5.

[28] H. Q. Nguyen, Global weak solutions for generalized SQG in bounded domains, Anal. PDE 11 (2018), no. 4, 1029-1047, doi:10.2140/apde.2018.11.1029, results for msqg in general domains

[29] J. Pedlosky. Geophysical Fluid Dynamics. Second Edition, Springer-Verlag, New York, 1987, doi:10.1007/978-1-4612-4650-3.

[30] S. Resnick, Dynamical Problems in Nonlinear Advective Partial Differential Equations, Ph. D. thesis University of Chicago, available at https://search.proquest. com/docview/304242616? accountid=14527.

[31] J. L. Rodrigo, On the evolution of sharp fronts for the quasi-geostrophic equation, Comm. Pure Appl. Math. 58 (2005), no. 6, 821-866, doi:10.1002/cpa.20059. 
[32] S. Schochet, The weak vorticity formulation of the 2-d euler equations and concentration-cancellation, Communications in Partial Differential Equations 20 (1995), no. 5-6, 1077-1104, doi:10.1080/03605309508821124.

[33] K. S. Smith, G. Boccaletti, C. C. Henning, I. Marinov, C. Y. Tam, I. M. Held, G. K. Vallis, Turbulent diffusion in the geostrophic inverse cascade, J. Fluid Mech. 469 (202), 13-48, doi:0.1017/S002211200200176.

[34] J. Wu, Solutions of the 2D quasi-geostrophic equation in Hölder spaces, Nonlinear Analysis 62 (2005), 579-594, doi:10.1016/j.na.2005.03.053. 